\title{
Vibrational behavior of bcc Cu-based shape-memory alloys close to the martensitic transition
}

\author{
Antoni Planes, Lluís Mañosa, and Eduard Vives \\ Departament d'Estructura i Constituents de la Matèria, Universitat de Barcelona, \\ Diagonal 647, Facultat de Física, E-08028 Barcelona, Catalonia, Spain
}

(Received 14 April 1995)

\begin{abstract}
Experimental data from ultrasonic and inelastic neutron scattering measurements are analyzed for different families of $\mathrm{Cu}$-based shape-memory alloys. It is shown that the transition occurs at a value, independent of composition and alloy family, of the ratio between the elastic constants associated with the two shears necessary to accomplish the lattice distortion from the bcc to the close-packed structure. The zone boundary frequency of the TA2[110] branch evaluated at the transition point $\left(T_{M}\right)$, weakly depends, for each family, on composition. A linear relationship between this frequency and the inverse of the elastic constant $C^{\prime}$, both quantities evaluated at $T_{M}$, has been found, in agreement with the prediction of a Landau model proposed for martensitic transformations.
\end{abstract}

\section{INTRODUCTION}

A number of metals and alloys crystallize into the open bcc structure and undergo, at a lower temperature, a phase transition towards a close-packed structure: this is the socalled martensitic transformation. ${ }^{1}$ It is a first-order diffusionless structural transition for which the path followed by the atoms can be suitably described in terms of a combination of two homogeneous shears: (110)[1 $1 \overline{1} 0]$ and $(1 \overline{1} 2)[\overline{1} 11] .^{2}$ Different stacking sequences are possible for the low-temperature phase, each of them being obtained by a static displacement corresponding to a phonon mode on the transverse $T A 2[110]$ branch, with a characteristic wavenumber $q$. For example, the $9 R$ (or $18 R$ ) structure is obtained for $q=\frac{2}{3} q_{\mathrm{ZB}}$ and the $2 H$, for $q=q_{\mathrm{ZB}}$. Neutron-scattering and ultrasonic experiments have shown that the whole $T A 2$ branch has very low energy and softens with decreasing temperature. The fact that this softening is not complete for any of the phonons (even at the transition temperature), evidences the first-order character of the transition. ${ }^{3}$

A simple dynamical instability of a phonon frequency reaching zero value at the transition temperature (soft-mode transition) is unsuitable to describe this kind of transition because of their marked first-order character. ${ }^{4}$ Beyond this approach, Lindgård and Mouritsen ${ }^{5}$ took the amplitude of the modulated distortion (associated with the stacking sequence of close-packed planes) as the main order parameter in a Landau theory, and they coupled it anharmonically to an homogeneous strain. They obtained that a low value (but finite) of the elastic constant $C^{\prime}$ associated with the homogeneous strain, gives the possibility of a first-order transition to occur. Generalized versions of their theory were later formulated by Gooding and Krumhans $1^{6,7}$ and by Gooding et al ${ }^{8}$

Examples of materials undergoing martensitic transformations are group-IV metals, some Ni-based alloys, and noblemetal based alloys. For a number of alloys, this kind of transition has received special attention because it is responsible for the technologically important shape-memory properties that they exhibit. ${ }^{1}$ This is the case of $\mathrm{Cu}$-based alloys (Cu-Zn, $\mathrm{Cu}-\mathrm{Zn}-\mathrm{Al}, \mathrm{Cu}-\mathrm{Al}-\mathrm{Be}, \mathrm{Cu}-\mathrm{Al}-\mathrm{Ni}$, etc.), which are the subject of investigation in the present work.

$\mathrm{Cu}$-based alloys are Hume-Rothery materials for which the phase stability is controlled by the average number of valence electrons per atom ratio $(e / a) .{ }^{9}$ The bcc phase is stable at high temperature, but the composition width of the stability region becomes smaller as temperature decreases, and, in many cases, a temperature (between 400 and 500 ${ }^{\circ} \mathrm{C}$ ) is reached at which the boundaries of this region meet each other. The electron concentration corresponding to this composition is close to $e / a=1.5$. Nevertheless, by suitable cooling, an ordered bcc phase can be retained at room temperature. The anomalous behavior of the TA2[110] branch mentioned before has clearly been observed for different families of $\mathrm{Cu}$-based alloys. ${ }^{10-12}$ The corresponding elastic constant $C^{\prime}\left[=\left(C_{11}-C_{12}\right) / 2\right]$, is also anomalously low; it softens with decreasing temperature but remains finite at the transition point $T_{M} \cdot{ }^{13,14}$ The low value of $C^{\prime}$ results in a high value of the elastic anisotropy $A=C_{44} / C^{\prime}$ for these alloys, which increases as $T_{M}$ is approached on cooling. ${ }^{15} \mathrm{~A}$ small dip at $q=(2 / 3) q_{\mathrm{ZB}}$ is always observed in the $T A 2[110]$ branch; it is independent of the modulation of the stacking sequence of the martensitic phase, being an evidence that the selection of the modulation of the lowtemperature phase cannot be exclusively determined by the anomaly in the phonon branch; rather, the structure chosen by the alloy depends on small details of the internal energy. ${ }^{3}$ On the other hand, it has been proved that the low energy of the TA2[110] vibrational modes provides the major contribution to the excess of entropy that stabilizes the bcc phase at high temperature. ${ }^{14,16}$

The aim of this paper is to analyze, in different families of composition related $\mathrm{Cu}$-based alloys, the pretransitional behavior of the elastic constants and the phonon modes within the frame of present Landau theories for martensitic transformations. We restrict ourselves to the minimal expansion that leads to a first-order transition resulting from a coupling between an homogeneous shear and a short-wavelength phonon. Our interest is to establish a link between the predictions derived from this model and the experimental observations for this class of alloys. The paper is organized as follows: in Sec. II we give a brief survey of the relevant 
Landau models formulated to account for the martensitic transformation. The experimental results for elastic constants and for phonon frequencies are presented in Sec. III. In Sec. IV, these experimental results are compared to the predictions given by Landau models. Finally, a summary of the most relevant points and conclusions are given in Sec. V.

\section{LANDAU-TYPE MODELS}

The simplest model containing the most significant physical features of the martensitic transformation is formulated in terms of two coupled order parameters: an homogeneous strain $e$ and the amplitude of a phonon mode $\Psi$; for a practical realization, they can be a (110) [1 $\overline{1} 0]$ shear and a phonon on the TA2[110] branch. ${ }^{5,17}$ Symmetry arguments allow the following terms in the Landau expansion of the free energy in terms of these two order parameters:

$$
F=\frac{1}{2} C^{\prime} e^{2}+\frac{1}{2} \omega^{2} \Psi^{2}+\frac{1}{4} \beta \Psi^{4}+\frac{1}{6} \gamma \Psi^{6}+\kappa e \Psi^{2},
$$

where the "spring constant" for the considered mode changes linearly with temperature:

$$
\omega^{2}=a\left(T-T_{c}\right),
$$

with $T_{c}$ the limit of stability of the undistorted ( $\Psi=0, e=0$ ) phase and $\beta$ and $\gamma$ positive constants. Minimization of the free energy with respect to the homogeneous deformation leads to the following effective free energy:

$$
F=\frac{1}{2} \omega^{2} \Psi^{2}+\frac{1}{4}\left(\beta-\frac{2 \kappa^{2}}{C^{\prime}}\right) \Psi^{4}+\frac{1}{6} \gamma \Psi^{6}
$$

When $\omega^{2} \rightarrow 0$, a pure soft-mode transition is possible at $T_{c}$. On the other hand, if $\left[\beta-\left(2 \kappa^{2} / C^{\prime}\right)\right]$ is negative, a firstorder transition can take place with a finite value of $\omega$. This term can only be negative for low enough values of $C^{\prime}$. The condition for this first-order transition to occur reads

$$
\omega\left(T_{M}\right)=\sqrt{\frac{3}{16 \gamma}}\left(\frac{2 \kappa^{2}}{C^{\prime}\left(T_{M}\right)}-\beta\right) .
$$

Taking into account the temperature dependence of $\omega$ (2), the equilibrium transition temperature $T_{M}$ is given by

$$
T_{M}=\frac{3 \kappa^{4}}{4 \gamma a}\left[\frac{\beta}{2 \kappa^{2}}-\frac{1}{C^{\prime}\left(T_{M}\right)}\right]^{2}+T_{c} .
$$

Detailed and more general formulations have been proposed to describe the transition from the bcc phase to different close-packed phases $(7 R$ and $9 R))^{7,6}$ Also the role played by distortions that do not affect the symmetry of the martensitic phase has been analyzed for the bcc-to- $9 R$ transition. ${ }^{8}$ The effect of these non-symmetry-breaking order parameters (associated with the elastic constant $C_{44}$ and with the bulk modulus $B$ ) is the reduction of the energy barriers between the two phases.

\section{EXPERIMENTAL RESULTS}

In this section we present results from ultrasonic and inelastic neutron-scattering measurements performed on differ-

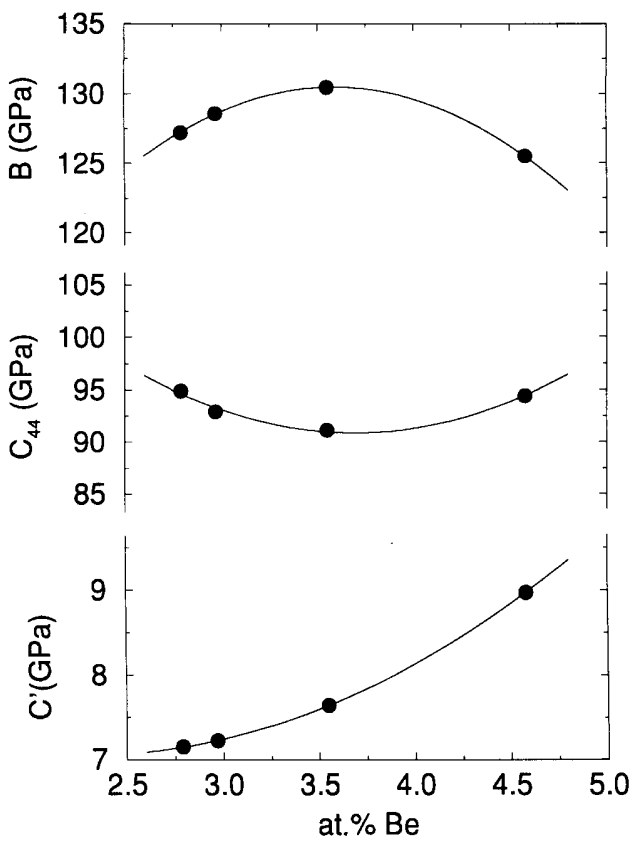

FIG. 1. Elastic moduli for a family of composition related $\mathrm{Cu}-\mathrm{Al}-\mathrm{Be}$ alloys. Data have been extracted from Ref. 14. Notice the low value of $C^{\prime}$ in comparison with the two other moduli. Lines are guides to the eye.

ent families of Cu-based alloys. In our study, we have preferentially used the data from those alloys in which both ultrasonic and neutron measurements have been performed on exactly the same sample. We also paid attention to the fact that measurements on the different samples were always performed under the same experimental conditions, so that the effect of possible systematic errors is minimized. The data used have been published in Refs. 14 and 18 for ultrasonic measurements and Ref. 12 for neutron measurements. It is not common to find published ultrasonic and neutron measurements performed on the same sample. In the case that data from only one technique were available, we used them to corroborate the findings obtained, in order to provide a unified picture for the vibrational behavior of $\mathrm{Cu}$-based alloys close to their martensitic transformation.

\section{A. The homogeneous strains}

bcc Hume-Rothery materials undergoing martensitic transformations have the common feature of having a high elastic anisotropy $A$, resulting from an anomalously low value of the elastic constant $C^{\prime}$. The low value for $C^{\prime}$ is illustrated in Fig. 1 for a family of composition related $\mathrm{Cu}-\mathrm{Al}-\mathrm{Be}$ alloys; the figure compares, for four different crystals, the elastic constants $B=(1 / 3)\left(C_{11}+2 C_{12}\right), C_{44}$ and $C^{\prime}$, which are associated with the strains in the irreducible representation for cubic symmetry. It is apparent from the figure that $B$ and $C_{44}$ show only a weak dependence on the concentration of $\mathrm{Be}$ (relative changes $\sim 5 \%$ ), while the dependence for $C^{\prime}$ is stronger (relative changes $\sim 25 \%$ ). It must be mentioned that the transition temperature $\left(T_{M}\right)$ for this family of alloys strongly depends on the composition; ${ }^{19}$ it linearly decreases as the atomic fraction of beryllium increases (a change of $200 \mathrm{~K}$ occurs for a variation of only 1 


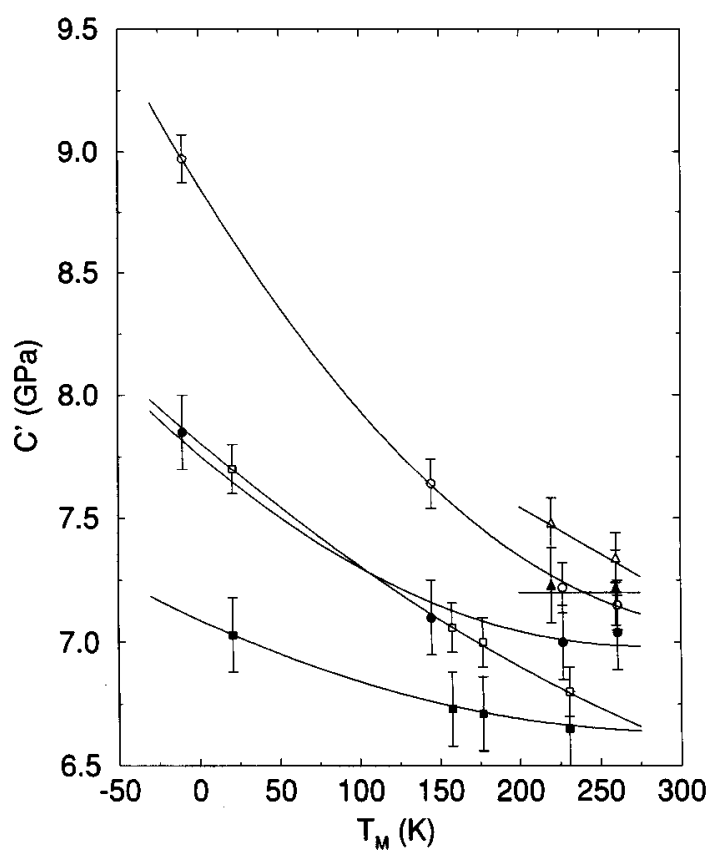

FIG. 2. Elastic constant $C^{\prime}$ for different families of Cu-based alloys as a function of their transition temperature $T_{M}$. Open symbols correspond to values at room temperature and filled symbols to values at the transition temperature. Data for $\mathrm{Cu}-\mathrm{Al}-\mathrm{Be}$ (circles) are from Ref. 14, for $\mathrm{Cu}-\mathrm{Al}-\mathrm{Ni}$ (triangles) from Ref. 18, and for $\mathrm{Cu}-$ $\mathrm{Zn}$-Al from Ref. 20. For $\mathrm{Cu}-\mathrm{Zn}-\mathrm{Al}$, the values at $T_{M}$ have been obtained by linear extrapolation using the same temperature dependence for all the alloys, extracted from Ref. 13. Lines are guides to the eye.

at. $\%$ of $\mathrm{Be}$ ). For this reason, $T_{M}$ must, in principle, be a good parameter to characterize each specific sample.

The stability of the bcc lattice for a shear on a (110) plane in the $[1 \overline{1} 0]$ direction is controlled by the elastic constant
$C^{\prime}$. Investigation, in different systems, of the value of $C^{\prime}$ at their corresponding transition temperature can result in valuable information on the elastic stability of the bcc lattice. In Fig. 2 we compare the values of $C^{\prime}$ at room temperature (open symbols) and at the corresponding transition temperature (filled symbols) for crystals pertaining to different families of $\mathrm{Cu}$-based alloys (data for $\mathrm{Cu}-\mathrm{Zn}-\mathrm{Al}$ are from Ref. 20). These values are tabulated in Table I. All families exhibit similar behavior for $C^{\prime}$ at room temperature: it decreases on increasing $T_{M}$. It could be expected that the softening of $C^{\prime}$ with temperature could result in the same value for this quantity at $T_{M}$ for all crystals in a family. Inspection of Fig. 2 shows that this is not the case, since for each family, $C^{\prime}$ at $T_{M}$ still decreases with $T_{M}$ but with a weaker dependence (notice the enlarged scale of the figure).

Until now we have only considered the elastic response of the lattice to a (110)[1 $\overline{1} 0]$ shear. As previously mentioned, a second homogeneous shear, $(1 \overline{1} 2)[\overline{1} 11]$, is needed in order to accomplish the lattice distortion from the bcc structure to a close-packed one. In order to elucidate the relevance of these two shears in comparison to the response to all other elastic distortions, it is convenient to obtain the velocity surfaces of the elastic waves. These surfaces are obtained from the nontrivial solutions of Christoffel equations for a system with cubic symmetry (see, for example, Ref. 21). Figure 3 shows the (110) cross section of the velocity surfaces for a $\mathrm{Cu}_{0.741} \mathrm{Al}_{0.231} \mathrm{Be}_{0.028}$ crystal, obtained from the measured values of the elastic constants $C_{L}, C_{44}$, and $C^{\prime}$, at room temperature. It is apparent from the figure that there are two modes for which the surface velocity displays minimum values, indicating that the lattice is softer for the mode (1 $\overline{1} 0)$ [110] (corresponding to the elastic constant $C^{\prime}$ ) and for an elastic wave close to the pure mode $(1 \overline{1} 2)[\overline{1} 11]$. It is worth remembering that these two modes are precisely the shears necessary to bring the bcc structure to the close-

TABLE I. Transition temperature $\left(T_{M}\right)$; shear elastic constant at room temperature $\left[C^{\prime}\left(T_{r}\right)\right]$ and at the transition temperature $\left[C^{\prime}\left(T_{M}\right)\right]$; special mode constant at room temperature $\left[C_{s}\left(T_{r}\right)\right]$ and at the transition temperature $\left[C_{s}\left(T_{M}\right)\right]$; elastic anisotropy at room temperature $\left[A\left(T_{r}\right)\right]$ and at the transition temperature $\left[A\left(T_{M}\right)\right]$; zone boundary frequency, at $360 \mathrm{~K}\left[\omega_{\mathrm{ZB}}(\mathrm{T})\right]$ and at the transition temperature $\left[\omega_{\mathrm{ZB}}\left(T_{M}\right)\right]$; and limiting temperature of the stability of the bcc phase $\left(T_{c}\right)$, for different $\mathrm{Cu}$-based alloys. Temperatures are given in $\mathrm{K}$, elastic constants in GPa, and frequencies in meV.

\begin{tabular}{|c|c|c|c|c|c|c|c|c|c|c|c|}
\hline Alloy & $T_{M}^{\mathrm{a}}$ & $C^{\prime}\left(T_{r}\right)$ & $C^{\prime}\left(T_{M}\right)$ & $C_{s}\left(T_{r}\right)$ & $C_{s}\left(T_{M}\right)$ & $A\left(T_{r}\right)$ & $A\left(T_{M}\right)$ & $\omega_{\mathrm{ZB}}(T)$ & $\omega_{\mathrm{ZB}}\left(T_{M}\right)$ & $T_{c}$ & Ref. \\
\hline $\mathrm{Cu}_{0.741} \mathrm{Al}_{0.231} \mathrm{Be}_{0.028}$ & 261 & 7.15 & 7.04 & 25.91 & 26.06 & 13.27 & 13.65 & 5.54 & 5.27 & -730 & 12,14 \\
\hline $\mathrm{Cu}_{0.742} \mathrm{Al}_{0.228} \mathrm{Be}_{0.029}$ & 228 & & & & & & & 5.61 & 5.23 & -751 & 12,14 \\
\hline $\mathrm{Cu}_{0.740} \mathrm{Al}_{0.230} \mathrm{Be}_{0.030}$ & 227 & 7.22 & 7.00 & 26.63 & 25.92 & 12.87 & 13.62 & 5.72 & 5.34 & -792 & 12,14 \\
\hline $\mathrm{Cu}_{0.737} \mathrm{Al}_{0.227} \mathrm{Be}_{0.036}$ & 145 & 7.64 & 7.10 & 25.67 & 26.16 & 11.83 & 13.50 & 5.76 & 5.16 & -805 & 12,14 \\
\hline $\mathrm{Cu}_{0.731} \mathrm{Al}_{0.223} \mathrm{Be}_{0.046}$ & -10 & 8.97 & 7.85 & 27.29 & 28.36 & 10.52 & 13.36 & & & & 14 \\
\hline $\mathrm{Cu}_{0.686} \mathrm{Al}_{0.276} \mathrm{Ni}_{0.038}$ & 260 & 7.34 & 7.22 & 26.09 & 26.26 & 12.60 & 13.40 & 5.27 & 4.96 & -618 & 12,18 \\
\hline $\mathrm{Cu}_{0.682} \mathrm{Al}_{0.280} \mathrm{Ni}_{0.038}$ & 220 & 7.48 & 7.23 & 26.31 & 26.67 & 13.06 & 13.44 & 5.38 & 4.97 & -663 & 12,18 \\
\hline $\mathrm{Cu}_{0.666} \mathrm{Zn}_{0.189} \mathrm{Al}_{0.145}$ & 168 & & & & & & & 5.24 & 4.67 & -611 & 12 \\
\hline $\mathrm{Cu}_{0.677} \mathrm{Zn}_{0.193} \mathrm{Al}_{0.130}$ & 274 & & & & & & & 5.28 & 5.02 & -626 & 10 \\
\hline $\mathrm{Cu}_{0.619} \mathrm{Zn}_{0.243} \mathrm{Al}_{0.138}$ & -120 & & & & & & & 6.52 & 5.25 & -1104 & 26 \\
\hline $\mathrm{Cu}_{0.68} \mathrm{Zn}_{0.16} \mathrm{Al}_{0.16}$ & 231 & 6.80 & 6.65 & & & 12.91 & 13.56 & & & & 20 \\
\hline $\mathrm{Cu}_{0.66} \mathrm{Zn}_{0.20} \mathrm{Al}_{0.14}$ & 177 & 7.00 & 6.71 & & & 12.40 & 13.57 & & & & 20 \\
\hline $\mathrm{Cu}_{0.66} \mathrm{Zn}_{0.21} \mathrm{Al}_{0.13}$ & 158 & 7.06 & 6.73 & & & 11.90 & 13.34 & & & & 20 \\
\hline $\mathrm{Cu}_{0.63} \mathrm{Zn}_{0.26} \mathrm{Al}_{0.11}$ & 21 & 7.70 & 7.03 & & & 10.90 & 13.34 & & & & 20 \\
\hline
\end{tabular}

${ }^{\mathrm{a}}$ Negative values correspond to linear extrapolated values as a function of composition. 


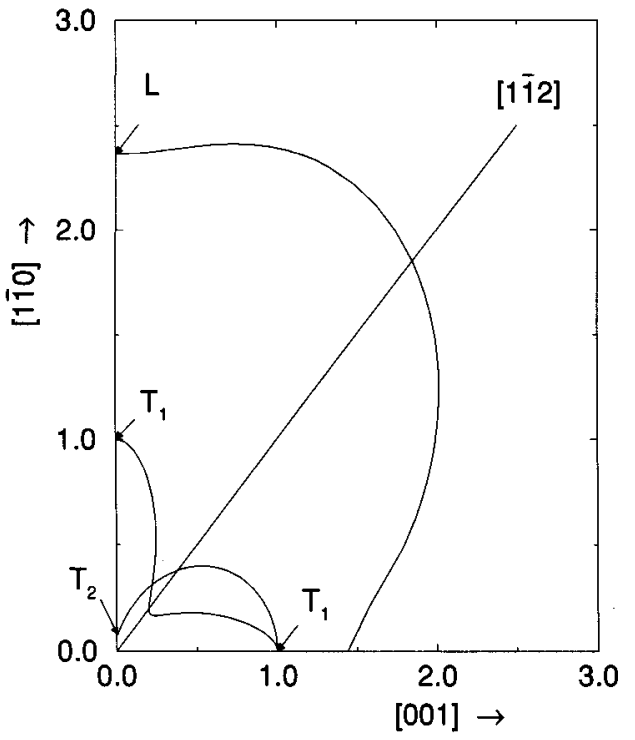

FIG. 3. (110) cross section of the square velocity surfaces in units of $C_{44} / \rho$ for a $\mathrm{Cu}_{0.741} \mathrm{Al}_{0.231} \mathrm{Be}_{0.028}$ crystal. They have been computed using the room-temperature values of the elastic constants published in Ref. 14. $L, T_{1}$, and $T_{2}$ correspond to the pure modes associated to $C_{L}, C_{44}$, and $C^{\prime}$, respectively.

packed one. The elastic constant $C_{s}$ associated to this second mode (termed by Nagasawa, Nakanishi, and Enami ${ }^{22}$ special mode) is given by

$$
C_{s}=C^{\prime}+\frac{1}{2} \chi\left(C_{44}-C^{\prime}\right)
$$

with

$$
\chi=2\left(C_{11}+C_{12}\right) /\left(3 C_{11}+5 C_{12}+2 C_{44}\right) .
$$

It is interesting to investigate the behavior of this special mode as the crystal approaches its transition temperature. The temperature dependence of $C_{s}$ has been obtained for $\mathrm{Cu}-\mathrm{Al}-\mathrm{Be}$ and $\mathrm{Cu}-\mathrm{Al}-\mathrm{Ni}$ alloys, and a typical example for the $\mathrm{Cu}_{0.737} \mathrm{Al}_{0.227} \mathrm{Be}_{0.036}$ crystal is presented in Fig. 4. This elastic constant shows no anomalous temperature dependence in the sense that it increases as temperature is lowered. All Cubased samples investigated showed similar temperature dependence. It is worth noting that $C_{s}$ for $\mathrm{Cu}$-based alloys behaves differently than it does for $\mathrm{Au}-\mathrm{Ag}-\mathrm{Cd}$ and $\mathrm{Ni}-\mathrm{Al}$ alloys for which a softening with decreasing temperature has been reported. ${ }^{22}$ The values of $C_{s}$ at room temperature and at the transition temperature for $\mathrm{Cu}$-based alloys, are given in Table I.

Under the central force approximation, and in the case of large anisotropy, $\chi$ turns out to be a constant of value 1/5. We have computed $\chi$ using Eq. (7) together with the data for the elastic constants at room temperature for $\mathrm{Cu}-\mathrm{Al}-\mathrm{Be}$ and $\mathrm{Cu}-\mathrm{Al}-\mathrm{Ni}$; the value found for all these crystals is very similar, averaging 0.215 \pm 0.002 . Moreover, in all cases this value changes with temperature less than $1 \%$ between room temperature and $T_{M}$. Actually, it appears that $C_{s}$ is in a very good approximation, a simple linear combination of the two elastic constants $C^{\prime}$ and $C_{44}$ [see Eq. (6)]. Hence, the mea-

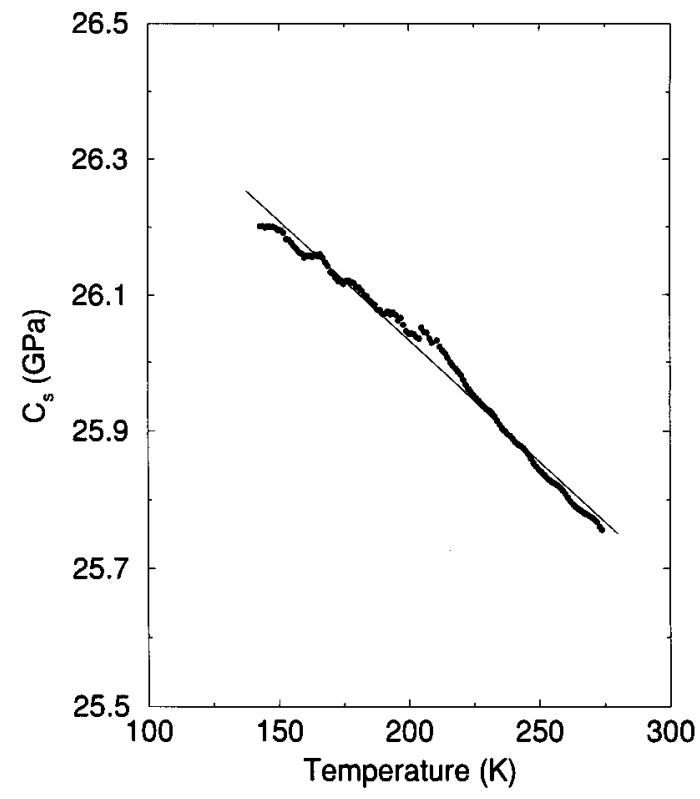

FIG. 4. Temperature dependence of the elastic constant $C_{s}$ associated with the special mode for a $\mathrm{Cu}_{0.737} \mathrm{Al}_{0.227} \mathrm{Be}_{0.036}$ crystal. It has been computed using data from Ref. 14.

sured increase in $C_{s}$ on cooling, arises from an increase in $C_{44}$, which is not completely compensated by a decrease of $C^{\prime}$.

From Eq. (6) it is clear that the elastic anisotropy $A=C_{44} / C^{\prime}$ controls the ratio between the two relevant shears in a martensitic transformation,

$$
\frac{C_{s}}{C^{\prime}}=1+\frac{1}{2} \chi(A-1) .
$$

The opposite temperature behavior found for $C^{\prime}$ and $C_{s}$, leads to the speculation that the transition could occur when the elastic anisotropy reaches a given specific value. Indeed, a fixed value of $A$ at the transition temperature, in $\mathrm{Cu}$-based alloys was already suggested in a previous paper. ${ }^{14}$ With this idea in mind, we have evaluated $A$ at room temperature and at the transition temperature for the different $\mathrm{Cu}$-based families; the results are tabulated in Table I and summarized in Fig. 5. It shows up from the figure that $A$ at $T_{M}$ is constant (with a value 13.5) within the experimental uncertainity for all $\mathrm{Cu}$-based alloys. This result has also been reported very recently by Romero and Pelegrina, ${ }^{23}$ who have also shown that $A$ does not depend on the electron concentration. Another important fact to remark from this figure is that all the data points for $A$ at room temperature collapse on the same curve; notice that this was not the case for $C^{\prime}$ (see Fig. 2), for which each family had its own curve. These findings reveal the important role of the second shear in the analysis of the instability of the bcc towards close-packed phases. Until now, to our knowledge, there is no theory predicting a constant value of $A$ at the transition temperature.

\section{B. The inhomogenous deformations}

In this section we analyze the lattice response to the inhomogeneous deformation associated with the martensitic transformation. This inhomogeneous deformation corresponds to a transverse $T A 2[110]$ phonon with a wave num- 


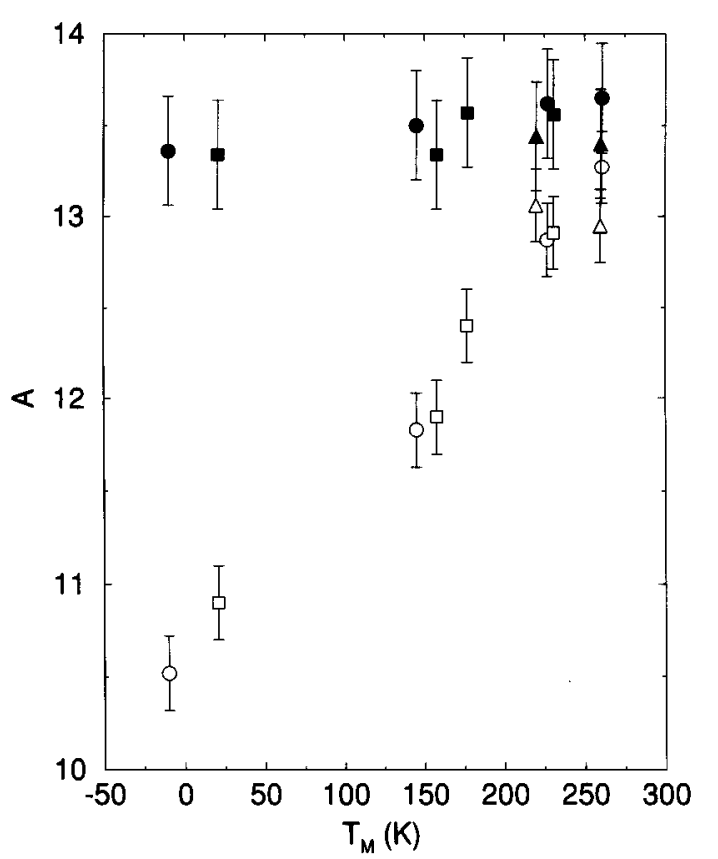

FIG. 5. Elastic anisotropy $A$ for different families of $\mathrm{Cu}$-based alloys as a function of their transition temperature $T_{M}$. Open symbols correspond to values at room temperature and filled symbols, to values at the transition temperature. Data for $\mathrm{Cu}-\mathrm{Al}-\mathrm{Be}$ (circles) are from Ref. 14, for $\mathrm{Cu}-\mathrm{Al}-\mathrm{Ni}$ (triangles) from Ref. 18, and for $\mathrm{Cu}-\mathrm{Zn}-\mathrm{Al}$ (squares) from Ref. 20. For $\mathrm{Cu}-\mathrm{Zn}-\mathrm{Al}$, the values at $T_{M}$ have been obtained by linear extrapolation using the same temperature dependence for all the alloys, extracted from Ref. 13.

ber characteristic for each sequence of the close-packed planes. Neutron measurements on Cu-based alloys ${ }^{24,12}$ have shown that the whole TA2 branch has low energy, and regardless of the close-packed structure of the low-temperature phase, there is always a small dip at $q=(2 / 3) q_{\mathrm{ZB}}$. The fact that this dip is also present in samples transforming to $2 \mathrm{H}$ indicates that it is not a signature of the structure of the low-temperature phase. ${ }^{3,12}$ On cooling, this dip does not become more pronounced [as occurs in other martensitic materials such as $\mathrm{Ni}-\mathrm{Al}$ (Ref. 25)], but it is the whole TA2 branch that softens. In addition, the $T A 2[110]$ branch is rather flat for high values of $q$. For all these reasons, we have used, for convenience, the zone boundary frequency $\omega_{\mathrm{ZB}}$ to analyze the lattice response to the inhomogeneous deformation.

In Fig. 6 we have plotted $\omega_{\mathrm{ZB}}^{2}$ at $370 \mathrm{~K}$ (open symbols), and at $T_{M}$ (filled symbols) for $\mathrm{Cu}-\mathrm{Al}-\mathrm{Be}$ (circles), and $\mathrm{Cu}-\mathrm{Al}-\mathrm{Ni}$ (triangles). The values at the transition temperature are obtained by a linear extrapolation explained in the following section. These values are also listed in Table I. The softening of $\omega_{\mathrm{ZB}}^{2}$ with lower temperature shows up in the figure by a lower value at $T_{M}$ than at room temperature. A dependence on $T_{M}$ is observed for $\omega_{\mathrm{ZB}}^{2}$ at room temperature; the corresponding values at $T_{M}$ seem to be closer for alloys in the same family, and a dependence with $T_{M}$ (the line in the figure) will be discussed in the next section. We have included in the inset data reported by different authors for $\mathrm{Cu}-\mathrm{Zn}-\mathrm{Al},{ }^{10,12,26}$ with their $T_{M}$ spread in a broad range. The measurements for the different samples have been performed on different diffractometers, and therefore comparison of the data can be affected by considerable systematic errors; even

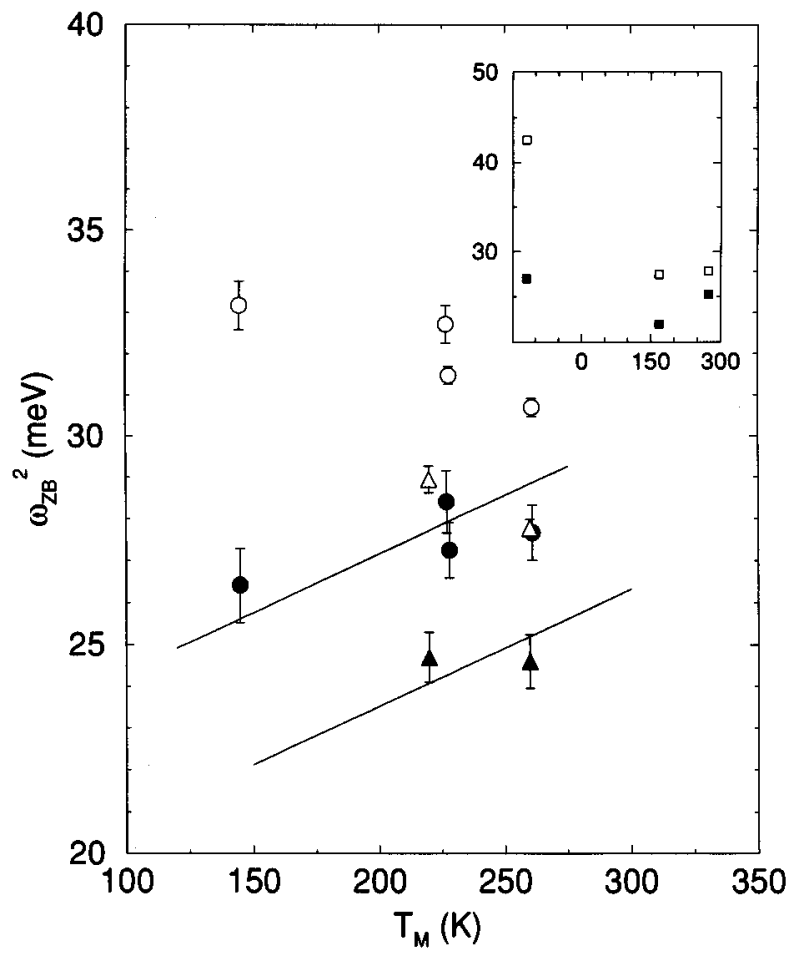

FIG. 6. Square of the frequency of the zone boundary TA2[110] phonon for different families of $\mathrm{Cu}$-based alloys as a function of their transition temperature. Open symbols correspond to values at $370 \mathrm{~K}$ and filled symbols to values at the transition temperature. Data for $\mathrm{Cu}-\mathrm{Al}-\mathrm{Be}$ (circles) and $\mathrm{Cu}-\mathrm{Al}-\mathrm{Ni}$ (triangles) are from Ref. 12. For all the alloys, the values at $T_{M}$ have been obtained by linear extrapolation with slope $0.028 \mathrm{meV}^{2} \mathrm{~K}^{-1}$. Lines correspond to $\omega_{\mathrm{ZB}}^{2}=a\left(T_{M}-T_{c}\right)$ with $a=0.028 \mathrm{meV}^{2} \mathrm{~K}^{-1}$ and $T_{c}=-770 \mathrm{~K}$ for $\mathrm{Cu}-\mathrm{Al}-\mathrm{Be}$ and $T_{c}=-640 \mathrm{~K}$ for $\mathrm{Cu}-\mathrm{Al}-\mathrm{Ni}$. Results for $\mathrm{Cu}-\mathrm{Zn}-\mathrm{Al}$ (squares) are shown in the inset; data are from Refs. 10,12 , and 26 , and we also have used the same slope to obtain values at $T_{M}$ and at $370 \mathrm{~K}$ (for those cases for which measurements were not performed at this temperature).

so, the points shown in the inset confirm the afore-mentioned behavior for $\omega_{\mathrm{ZB}}^{2}$ at room temperature and at $T_{M}$.

\section{COMPARISON OF EXPERIMENTAL RESULTS WITH THE PREDICTIONS OF THE LANDAU MODEL}

We are now committed to the analysis of the experimental results, in terms of the predictions of the Landau model outlined in Sec. II. The model includes two order parameters: the amplitude $\Psi$ of a phonon mode and an homogeneous strain $e$. The inverse of the susceptibilities $\left(\omega^{2}\right.$ and $\left.C^{\prime}\right)$ associated with these order parameters must be identified to measurable quantities. As previously mentioned, all phonons in the $T A 2[110]$ branch behave similarly with temperature, and, therefore, $\omega$ can be identified with any of the shortwavelength phonons. Nevertheless, in order to have results comparable for different alloys, $\omega$ must be taken at the same $q$ for all the samples (independently of the specific stacking sequence of the low temperature phase). On the other hand, it is worthwile to remark that the elastic shear associated with the special mode is not included in the model.

Equation (4) predicts a linear behavior of $\omega$ with the inverse of $C^{\prime}$, both quantities evaluated at $T_{M}$. We have 


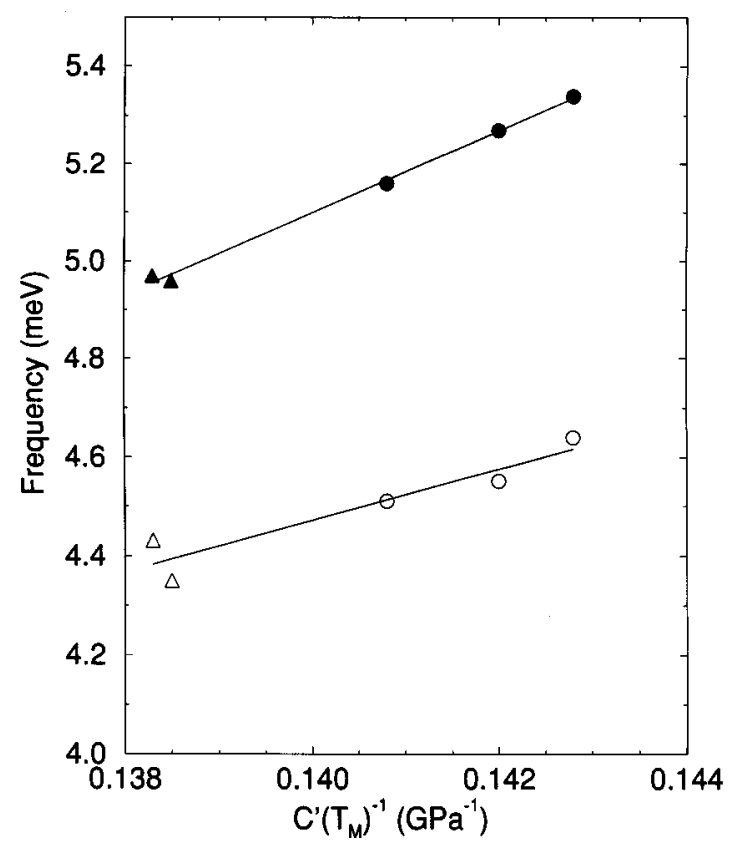

FIG. 7. Frequency at $q=(2 / 3) q_{\mathrm{ZB}}\left(\omega_{2 / 3}\right)$ (open symbols) and at the zone boundary $\left(\omega_{\mathrm{ZB}}\right)$ (filled symbols) in the TA2[110] phonon branch as a function of the inverse of the elastic constant for different families of $\mathrm{Cu}$-based alloys. Both quantities are evaluated at the transition temperature. Data for $\mathrm{Cu}-\mathrm{Al}-\mathrm{Be}$ (circles) are from Refs. 14 and 12 and for Cu-Al-Ni (triangles) from Refs. 12 and 18.

checked the validity of this prediction by plotting in Fig. 7 $\omega_{\mathrm{ZB}}$ (filled symbols) and $\omega_{2 / 3}\left(\omega\right.$ at $q=(2 / 3) q_{\mathrm{ZB}}$ ) (open symbols) vs $\mathrm{C}^{\prime-1}$, for $\mathrm{Cu}-\mathrm{Al}-\mathrm{Be}$ (circles) and $\mathrm{Cu}-\mathrm{Al}-\mathrm{Ni}$ (triangles). In both cases, a linear behavior is observed in concordance with the model. In the following, we will identify the model parameter $\omega$ with the zone boundary frequency $\omega_{\mathrm{ZB}}$. The fact that data points for different families all lie in the same straight line, leads to the conclusion that $\beta / \sqrt{\gamma}$ and $\kappa^{2} / \sqrt{\gamma}$ are independent of composition and alloy system [see Eq. (4)]. A linear fit renders $\kappa^{2} / \sqrt{\gamma}=97.8 \mathrm{meV} \mathrm{GPa}$ and $\beta / \sqrt{\gamma}=15.6 \mathrm{meV}$.

We now turn our attention to the remaining parameters: the slope $a$ of the temperature dependence of $\omega_{\mathrm{ZB}}^{2}$ and the temperature $T_{c}$ for which $\omega_{\mathrm{ZB}}$ goes to zero (limit of stability of the bcc phase). They can be obtained from the measured temperature dependence of $\omega_{\mathrm{ZB}}^{2}$ plotted in Fig. 8 for $\mathrm{Cu}-\mathrm{Al}-\mathrm{Be}$ (cricles), $\mathrm{Cu}-\mathrm{Al}-\mathrm{Ni}$ (triangles), and $\mathrm{Cu}-\mathrm{Zn}-\mathrm{Al}$ (squares). Within the experimental uncertainties, the data for the different crystals can consistently be fitted by straight lines with the same slope $a=0.028 \mathrm{meV}^{2} \mathrm{~K}^{-1}$, for all families. ${ }^{27}$ Only for the $\mathrm{Cu}_{0.619} \mathrm{Zn}_{0.243} \mathrm{Al}_{0.138}$ crystal (with $T_{M}=-120 \mathrm{~K}$ ) does this fit seem to be inappropriate. There is a different line for each composition, and extrapolation of a linear behavior down to $\omega_{\mathrm{ZB}}^{2}=0$ results in a different value for $T_{c}$ for each composition. The values obtained for $T_{c}$ for the different alloys are given in Table I. It is worth noticing that for all the alloys investigated $T_{c}$ is negative, thus showing the impossibility of a second-order soft-mode transition (dynamical instability of the mode frequency) to occur. The values found for $\mathrm{Cu}$-based alloys are lower than the values reported for $\mathrm{Ni}_{62.6} \mathrm{Al}_{37.5} \quad(=-30 \quad \mathrm{~K}) \quad$ and $\mathrm{Ni}_{63.9} \mathrm{Al}_{36.1}(=100 \mathrm{~K}) ;{ }^{28}$ and for $\mathrm{Ti}(\sim-150 \mathrm{~K})$ and $\mathrm{Zr}$

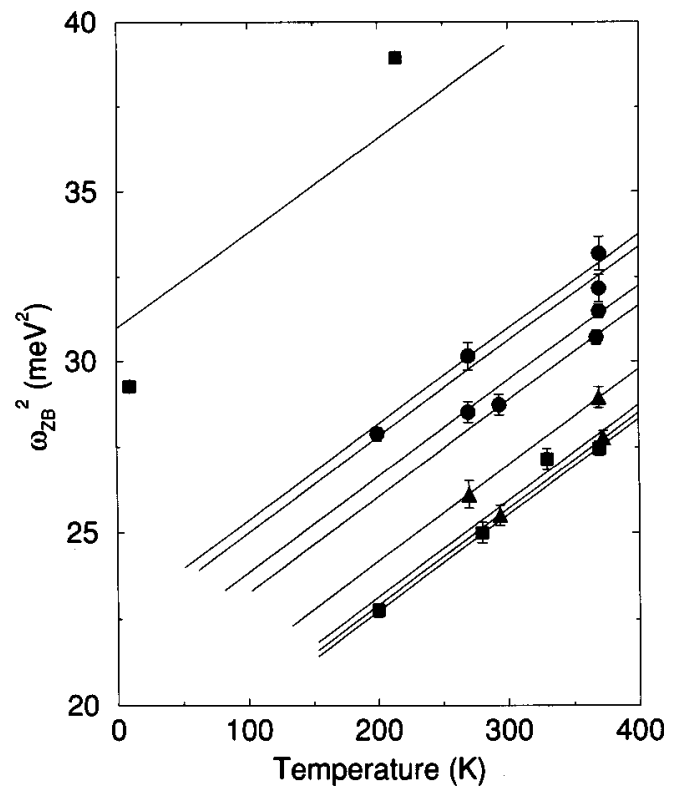

FIG. 8. Temperature dependence of the square of the frequency of the zone boundary TA2[110] phonon for different families of $\mathrm{Cu}$-based alloys. Data for $\mathrm{Cu}-\mathrm{Al}-\mathrm{Be}$ (circles) and $\mathrm{Cu}-\mathrm{Al}-\mathrm{Ni}$ (triangles) are from Ref. 12. Data for $\mathrm{Cu}-\mathrm{Zn}$-Al (squares) are from Refs. 10, 12, and 26. Notice that straight lines with a unique slope of $0.028 \mathrm{meV}^{2} \mathrm{~K}^{-1}$ fit consistently the experimental points.

$(\sim 60 \mathrm{~K}) .{ }^{29} T_{c}$ are obtained by extrapolation down to a value far away from the experimental points, and hence they can be affected by considerable error; nevertheless, data for each family of alloys distribute around a given value (a large scatter is obtained for $\mathrm{Cu}-\mathrm{Zn}-\mathrm{Al})$. In order to proceed further in our comparison with the predictions of the Landau model, the dependence of $T_{c}$ with composition should be known. Actually, there is not enough experimental information to determine this dependence reliably, and, hence, for alloys in a restricted range of compositions, it seems to be valid to assume a constant (average) value for each family. In other words, in the following, we will assume $a$ independent of both alloy family and composition, and $T_{c}$ independent of composition but different for each family. By averaging the values in Table I, we obtain $T_{c}=-770 \mathrm{~K}$ for $\mathrm{Cu}-\mathrm{Al}-\mathrm{Be}$; $T_{c}=-640 \mathrm{~K}$ for $\mathrm{Cu}-\mathrm{Al}-\mathrm{Ni}$, and $T_{c}=-780 \mathrm{~K}$ for $\mathrm{Cu}-\mathrm{Zn}-\mathrm{Al}$. The assumption of a constant $T_{c}$ for each family, results in a linear dependence of $\omega_{\mathrm{ZB}}^{2}\left(T_{M}\right)$ with $T_{M}$ [see Eq. (2)], which has been plotted in Fig. 6. Within the experimental errors, the behavior is rather consistent with the experimental data. This finding shows that $\omega_{\mathrm{ZB}}$ at $T_{M}$ decreases with $T_{M}$.

Finally, we can now check the validity of Eq. (5), since all the parameters have already been evaluated for all alloy families. In Fig. 9, we have represented, the experimental values (points) of $T_{M}$ as a function of $\left[\beta / 2 \kappa^{2}-1 / C^{\prime}\left(T_{M}\right)\right]^{2}$ with $\beta / 2 \kappa^{2}=0.080 \mathrm{GPa}^{-1}$, together with the result of Eq. (5) with the parameters evaluated previously for $\mathrm{Cu}-\mathrm{Al}-\mathrm{Be}$ and $\mathrm{Cu}-\mathrm{Al}-\mathrm{Ni}$ (solid lines). For $\mathrm{Cu}-\mathrm{Zn}$ $\mathrm{Al}$, the line plotted is a linear fit with the same slope as for the other alloys, and $T_{c}$ as a free parameter; in this case the best fit is obtained for $T_{c}=-1045 \mathrm{~K}$. This value is slightly larger than the average value $T_{c}=-780 \mathrm{~K}$ but still consistent considering the scatter of the data in Table I. It is also 


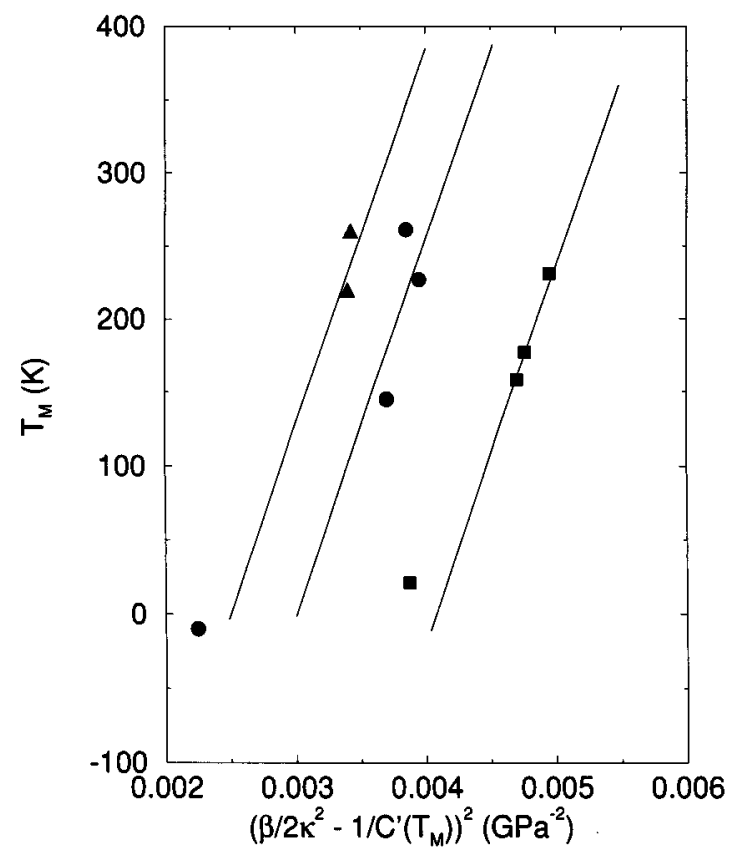

FIG. 9. Relationship between the transition temperature $T_{M}$ and the inverse of the elastic constant $C^{\prime}$ at the transition temperature for different families of $\mathrm{Cu}$-based alloys. Data for $\mathrm{Cu}-\mathrm{Al}-\mathrm{Be}$ (circles) are from Ref. 14, for Cu-Al-Ni (triangles) from Ref. 18 and for $\mathrm{Cu}-\mathrm{Zn}-\mathrm{Al}$ (squares) from Ref. 20. Lines correspond to Eq. (5), as explained in the text.

worth mentioning that the values for the alloys with a very low $T_{M}$ lie quite far from the fits. Such discrepancies could be expected, since, as already mentioned, the temperature dependence of $\omega_{\mathrm{ZB}}^{2}$ is different for alloys with $T_{M}$ significantly different from the rest of the alloys (see Fig. 8). That is, the hypothesis of a constant temperature softening of $\omega^{2}$ (constant $a$ ) is probably not too adequate for alloys with transition temperatures spreading over a very broad range.

\section{SUMMARY AND CONCLUSIONS}

In this paper, we have analyzed a set of experimental data from ultrasonic and inelastic neutron-scattering measure- ments, for three families of Cu-based shape-memory alloys. It has been shown that, in order to account for the martensitic transition, the two shears that bring the bcc structure to the close-packed one must be considered. In this sense, we have provided experimental evidence that the transition in $\mathrm{Cu}$ based alloys occurs at a fixed value of the ratio $C_{s} / C^{\prime}$ between the elastic constants associated to these shears, which results in a fixed value of the elastic anisotropy $A$ at the transition point. To reach this fixed value, an extra softening of $C^{\prime}$ is needed, owing to the fact that $C_{s}$ increases on cooling. It has also been shown that the zone boundary frequency of the TA2[110] branch, evaluated at the transition temperature is different for the different families of alloys, and, within each family, it shows a weak linear dependence on the transition temperature. The results have been analyzed in the framework of a Landau theory in which the first-order transition is possible caused by the anharmonic coupling between the homogeneous strain associated with $C^{\prime}$ and a short-wavelength phonon in the $T A 2[110]$ branch. In spite of its simplicity, the model is suitable to describe in a unified way, the transition for Cu-based alloys with transition temperatures in a relatively broad temperature range. This work is the first experimental evidence that the coupling between an homogeneous shear and a short-wavelength phonon is an essential mechanism to account for the martensitic transformation. It is worth noting that although the model does not include the second shear, associated with $C_{s}$, the experimental results behave as predicted by this model. This finding is an indication that the two shears are not independent; indeed the constancy of the elastic anistropy at the transition point is an experimental proof of the interdependence between the two shears. The effect of this second shear and other possible terms [as third-order terms in the homogeneous shear(s)], is to modify the specific values of the vibrational response (given by the phonon frequency and the elastic constant) at the transition point but not their qualitative behavior.

\section{ACKNOWLEDGMENTS}

We acknowledge J. Krumhansl and P.A. Lindgård for fruitful discussions and L. Delaey for a critical reading of the manuscript. This work received financial support from the CICyT (Spain), Project No. MAT95-0504.
${ }^{1}$ L. Delaey, Materials Science and Technology, Vol. 5 Phase Transformations in Materials, edited by P. Haasen (VCH, Weinheim, 1991), p. 339.

${ }^{2}$ A. Nagasawa and Y. Morii, Mater. Trans. JIM 34, 855 (1993).

${ }^{3}$ W. Petry, J. Phys. IV (France) 5, C2-15 (1995).

${ }^{4}$ J.A. Krumhansl and R.G. Gooding, Phys. Rev. B 39, 3047 (1989).

${ }^{5}$ P.A. Lindgård and O.G. Mouritsen, Phys. Rev. Lett. 57, 2458 (1986).

${ }^{6}$ R.J. Gooding and J.A. Krumhansl, Phys. Rev. B 38, 1695 (1988).

${ }^{7}$ R.J. Gooding and J.A. Krumhansl, Phys. Rev. B 39, 1535 (1989).

${ }^{8}$ R.J. Gooding, Y.Y. Ye, C.T. Chang, K.M. Ho, and B.N. Harmon, Phys. Rev. B 43, 13626 (1991).

${ }^{9}$ T.B. Massalski and U. Mizutani, Prog. Mater. Sci. 22, 151 (1978).
${ }^{10}$ G. Guénin, S. Hautecler, R. Pynn, P.F. Gobin, and L. Delaey, Scripta Metal. 13, 429 (1979).

${ }^{11}$ Y. Morii and M. Iizumi, J. Phys. Soc. Jpn. 54, 2948 (1985).

${ }^{12}$ Ll. Mañosa, J. Zarestky, T. Lograsso, D.W. Delaney, and C. Stassis, Phys. Rev. B 48, 15708 (1993).

${ }^{13}$ G. Guénin, M. Morin, P.F. Gobin, W. Dejonghe, and L. Delaey, Scripta Metal. 11, 1071 (1977).

${ }^{14}$ A. Planes, Ll. Mañosa, J. Ortín, and D. Ríos-Jara, Phys. Rev. B 45, 7633 (1992).

${ }^{15}$ V. Verlinden and L. Delaey, Acta Metall. 36, 1771 (1988).

${ }^{16}$ Ll. Mañosa, A. Planes, J. Ortín, and B. Martínez, Phys. Rev. B 48, 3611 (1993).

${ }^{17}$ J.A. Krumhansl, Solid State Commun. 84, 251 (1992). 
${ }^{18}$ Ll. Mañosa, M. Jurado, A. Planes, J. Zarestky, T. Lograsso, and C. Stassis, Phys. Rev. B 49, 9969 (1994).

${ }^{19}$ D. Ríos-Jara, J. Ortín, Ll. Mañosa, A. Planes, S. Belkahla, and M. Morin, Proceedings of ICOMAT'92, Monterey, CA, 1992, edited by C. M. Wayman and J. Perkins (Monterey Institute for Advanced Studies, Monterey, 1993), p. 383.

${ }^{20}$ T. Suzuki, Y. Fujii, R. Kojima, and A. Nagasawa, Proceedings of ICOMAT'86, Nara, 1986 (The Japan Institute of Metals, Sendai, 1986), p. 874.

${ }^{21}$ A.G. Every, Phys. Rev. Lett. 42, 1065 (1979); M. Sato, R.D. Lowde, G.A. Saunders, and M.M. Hargreave, Proc. R. Soc. London, Ser. A 374, 115 (1981).

${ }^{22}$ A. Nagasawa, N. Nakanishi, and K. Enami, Philos. Mag. A 43, 1345 (1981).
${ }^{23}$ R. Romero and J.L. Pelegrina, Phys. Rev. B 50, 9046 (1994).

${ }^{24}$ G. Guénin, D. Ríos-Jara, M. Morin, L. Delaey, R. Pynn, and P.F. Gobin, J. Phys. (Paris) 43, C4-597 (1982).

${ }^{25}$ S.M. Shapiro, B.X. Yanh, G. Shirane, Y. Noda, and L.E. Tanner, Phys. Rev. Lett. 62, 1298 (1989).

${ }^{26}$ D. Abbé, R. Caudron, and R. Pynn, J. Phys. F 14, 1117 (1984).

${ }^{27} \mathrm{~A}$ value of $a=0.02 \mathrm{meV} / \mathrm{K}$ is obtained if $\omega_{2 / 3}$ is used instead of $\omega_{\mathrm{ZB}}$.

${ }^{28}$ This value has been reported by S.M. Shapiro, B.X. Yang, Y. Noda, L.E. Tanner, and D. Schryvers, Phys. Rev. B 44, 9301 (1991) by extrapolating the square of the frequency of the dip down to zero.

${ }^{29}$ We have obtained these values by extrapolating the temperature dependence of $\omega_{\mathrm{ZB}}^{2}$, reported in Ref. 3 . 